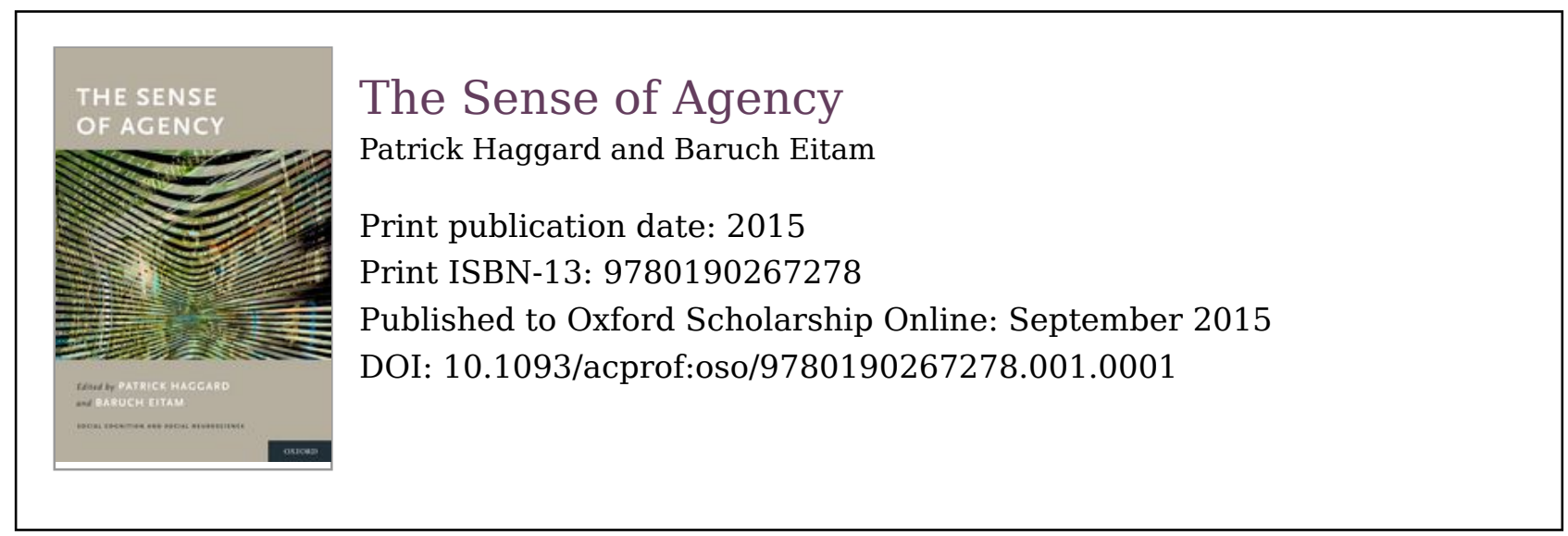

\title{
Sense of Agency and Its Disruption
}

\author{
Clinical and Computational Perspectives
}

Paul Fletcher

Aikaterini Fotopoulou

DOI:10.1093/acprof:oso/9780190267278.003.0016

\begin{abstract}
Keywords
Sense of agency-the feeling of being the author of one's actions-may be a critical component of one's sense of self and of one's interaction with the world. Insights from clinical and experimental neuropsychology, as well as cognitive and computational neuroscience, have provided complementary evidence that the sense of agency arises from the integration of an array of internal and external cues. These frameworks can help to explain how disruptions in one or more of these cues may result in altered experiences of agency. This chapter reviews these explanatory frameworks and shows how important and useful they have become in making sense of an array of clinical observations, from the disorders of control and agency that result from circumscribed brain damage to the widespread attenuation of agency that may characterize psychosis in which no clear brain lesion has been identified.
\end{abstract}

Keywords: experimental neuropsychology, cognitive, computational neuroscience, agency, brain damage, psychosis

\section{Introduction}

In this chapter, we examine the nature of sense of agency from a clinical and a computational perspective. Beginning with a consideration of the nature of the subjective experience of agency and the complexity of factors that may underlie, we discuss briefly the emergent models seeking to understand the processes that govern and shape it. In doing so, we point to certain areas of contention 
before taking these ideas forward into a discussion of agency in neuropsychiatry, focusing on agency in psychosis (notably delusions of control), Anosognosia for Hemiplegia and Anarchic Hand syndrome. In doing so, we will attempt to apply the insights afforded by the theoretical models of SoA and motor awareness that we consider. We believe such consideration may enhance the understanding of the pathogenesis of these disorders as well as the mechanisms underlying the SoA and motor awareness more generally.

We begin by considering the sense of agency and how it might be quantified.

What Is Sense of Agency and How Do We Measure It?

A fundamental aspect of our self-consciousness is the compelling feeling of authorship and control that accompanies and characterizes many of our (p.348) actions (Gallagher, 2000). We see ourselves as the wilful originators of our acts and, moreover, when an action is performed, we recognize ourselves as its prime controller. In the last two decades there has been a burgeoning body of neuroscientific and neurophilosophical literature on such notions of "agentive self-awareness" (Pacherie, 2007). Despite such progress, however, we are faced with inconsistency, complexity, and contention. In fact, most of the processes underlying our movements are unconscious; we seem to become aware only of a small proportion of them. Key facets of this limited and elusive agentive selfawareness include (1) the sense of agency (SoA), or the subjective feeling that we, and not somebody else, have caused and controlled an action and its effects (a narrow, heuristic definition of the SoA); (2) the subjective feeling that we are moving, or have just executed a movement (motor awareness); and (3) the awareness of our own intention or urge to act (ranging from "prior intentions," such as intending to go for a swim, to "intentions-in-action," such as intending to take a dive; Searle, 1983).

It should be noted that, despite such seemingly clear definitions and demarcations, these awareness concepts are themselves multifaceted. The relation between them is, at times, unclear, and their phenomenal contents are rather slippery. For example, in many, if not most, cases, we would not necessarily experience ongoing consciousness of initiating, executing, and controlling actions, yet we would argue with someone who denied that it was we who caused, executed, and controlled the actions in question. Under certain circumstances, healthy people may incorrectly attribute agency to actions that they do not really govern, or may fail to attribute agency to those that they do. One example of the former is "water-divining," in which movements of a rod, caused by the carrier, are actually attributed to the presence of some force exerted by a nearby body of water. Conversely, many people are unaware of the degree to which the movements of a cursor on a screen are actually assisted by the computer software, attributing it entirely to themselves (Fourneret \& Jeannerod, 1998; Sarrazin et al., 2008). Added to this is a degree of contention over what sorts of cues (internal or external) primarily engender and shape our 
SoA and how these may be integrated. The precise mechanisms for such integration and the nature of signals-both those originating internally and externally - that are critical to SoA are also unclear. Similarly, in the domain of motor awareness, we have the everyday belief that we are aware of the kinds of actions we execute as we perform them. In reality, so long as an expected action goal is achieved, we are largely unaware of precisely how we executed the movements involved, including the errors and adjustments we had to make on the way (Fourneret \& Jeannerod, 1998; Sarrazin et al., 2008). This implies that our normal subjective feeling of action execution is, to a degree at least, nonveridical and distinct from motor control. Thus, the precise mechanisms (p.349) by which mostly efficient and non-conscious processes of motor control give rise to our non-veridical and conscious feelings of movement execution remain unknown.

It is worth noting at the outset that successful scientific study of SoA and related awareness notions is obviously reliant on the degree to which such senses may be identified reliably and quantified accurately. Generally, subjective reports have been used-experimental participants are required to indicate their feeling that an action has been carried out or not and, if yes, whether it was they who caused it or some external agent. Such judgments may be binary (did I or didn't I initiate this movement?) or they may be rated in terms of the strength of the sense under consideration. There are other, more indirect, measures of both agency and motor awareness. While their indirectness may be disadvantageous, they do offer complementary quantitative measures of SoA and motor awareness, allowing subtle experimental assessments that might not otherwise be possible. One phenomenon that has been suggested to provide an indirect measure of agency is intentional binding, which is a temporal measure of the degree to which an action and an ensuing outcome are "bound" in time. The key finding with such measures is that, when an action is intentional, the actor perceives it to be closer in time to the outcome than it actually is. If the action is not internally generated but is produced by, for example, transcranial magnetic stimulation, the binding effect is lost, suggesting that it is peculiar to instances in which there is agency. This therefore offers the possibility that binding may be a reliable surrogate measure for agency. Interestingly, this binding effect appears to be produced not just by the presence of the action's outcome but also by the agent's prediction of the outcome (the perception of the time of action shifts, when there is a strong prediction of an outcome, even when that outcome does not actually ensue). Using combinations of subjective judgment of agency and intentional binding, it has been possible to explore the impact of external factors and expectations (Moore et al., 2013) on both explicit and implicit measures of SoA. The intentional binding approach has, moreover, proven useful and sensitive in studies of conditions such as schizophrenia in which agency is thought to be altered. 
Similarly, within the domain of motor awareness, one could trace at least two major distinctions, each with related empirical implications. First, as with other domains of awareness, there is the distinction between implicit and explicit indices. Explicit measures of awareness relate to conscious, subjective feelings and thoughts that are available for verbal report, while implicit ones relate to "knowledge that is expressed in task performance unintentionally and with little or no phenomenal awareness" (Schacter, 1990, p. 157). Thus, while motor awareness can be measured by verbal report, implicit measures include reaction times (Nardrone et al., 2007; Fotopoulou et al., 2010) or (p.350) choice of behavioral strategy (e.g., Cocchini et al., 2010; Moro et al., 2011). Second, there is a distinction between "on-line" (or "emergent") and "off-line" (or "anticipatory") motor awareness (Carruthers, 2008; Crosson et al., 1989; see also Tsakiris \& Fotopoulou, 2008). The former terms refer to the subjective feeling of moving in the moment or having just moved, while the latter refer to a more general expectancy and inference about one's ability to move and execute future actions, as for example when one is feeling able to reach a target. This distinction also necessitates different types of measurements, including, for example, confrontation tasks where participants are asked to execute movements and report on their experience versus estimation tasks where subjects are asked to estimate their future performance in given motor tasks. The exact relation between these facts of motor awareness, as well as between their respective measurements, remains currently unclear. However, studies in neuropsychiatric populations have demonstrated that such aspects of awareness may dissociate between and even within patients (Cocchini et al., 2010;

Fotopoulou et al., 2010; Moro et al., 2011), and hence they warrant separate and careful consideration.

We will return to examples of studies using both implicit and explicit measures of agency and action awareness in the context of neuropsychiatric disease. Before this, given some of the above contentions over the nature of motor agency and awareness, we will consider existing models of how SoA arises and what factors may shape it, including internal and external cues, as well as the levels of uncertainty and noise that modulate the impact of these cues. In considering these factors we will introduce computational models of agency appealing to optimal motor control, predictive coding, and active inference and discuss their respective contributions to our understanding of these fundamental aspects of the bodily self.

Internal and External Cues to Agency One simple distinction in models of SoA concerns the extent to which this sense emerges as a consequence of internal cues (for example, those concerned with sensorimotor experiences) or of external cues (for example, priming with cues suggestive that one is indeed the agent of action). We have reviewed this elsewhere (see Moore \& Fletcher, 2012) and it has been more extensively treated by Synofzik, Vosgerau, and Newen (2008). Indubitably, both forms of cue 
can engender SoA, even to the extent of producing a false SoA for an action for which one had not been responsible (Wegner \& Wheatley, 1999; Moore, Wegner \& Haggard, 2009). To be a little more specific, the internal cues providing a signal that a movement was agentic involve the conscious experience of the intention or the desire to move, as well as the proprioceptive and other sensory changes (p.351) signaling that a movement has indeed occurred. One suggestion, for example, is that a true sense of agency emerges when the predicted sensory/proprioceptive consequences (based on a "forward model" shaped by a computation of the actions required to achieve the goal or intention) closely match those consequences that actually ensue. A mismatch, it is suggested, may well designate a movement that was not intentional or under one's own control. This comparator view of the emergence of SoA will be considered briefly below, as well as models that reject this view of SoA arising from an absence of mismatch.

Perhaps less obviously, external cues may influence the experience of agency. Wegner and Wheatley (1999) pointed out that the experience of willed action relies on a tripartite experience connected with an internal thought about the act in question: specifically, that this thought is prior to the act, that it is consistent with this act, and that it alone can account for that act. In this respect, the SoA is a causal attribution, entailing the same criteria as if one were making a causal judgement about any two events. The experience of willing the action is a candidate cause, but not the only cause, of bodily movement. Such a perspective offers insights to erroneous SoA and also allows for the possibility that external factors may modulate the experience of action in a number of ways. To highlight this, Wegner and Wheatley elegantly demonstrated the impact of external cues by showing that, in a situation in which agency for an action (cessation of a continuous movement controlling a cursor on a screen) was ambiguous, the presence of a relevant, external, auditorily presented, prime word enhanced a participant's sense of agency for this action.

In short, therefore, SoA is shaped by both internal and external cues. This raises a question: If the agent receives an array of cues-both internal and externalpotentially relevant to agency, how might these be combined optimally? Moreover, what might be the outcome when cues are contradictory? We have previously speculated that, just as has been considered extensively for sensory cues (e.g., Ernst \& Bulthoff, 2004), cues to agency may be combined and integrated according to their estimated reliability or precision (Moore \& Fletcher, 2012). Put simply, if two cues offer information about agency, then they may be optimally combined by taking into account the precision (the inverse variance) of each and weighting them accordingly, cues estimated to be more precise being accorded a greater weighting. This optimal integration can be represented formally in terms of maximum likelihood estimation. A consequence of such integration is an overall reduction in variance or an enhancement of precision. As an example, in sensory processing, integration of visual and haptic 
information is likely to weight the former more heavily due to its (generally) superior precision.

Of course, as encapsulated by Bayes's Theorem, one critical factor that can be added to the maximum likelihood estimation to further optimize one's (p.352) estimation or conclusion is prior belief. Priors, according to Bayesian models, form a critical part of the equation and, in the face of noisy/imprecise internal or external cues, provide a way of more completely combining cues. This combination or integration is discussed more fully elsewhere (see, for example, Moore \& Fletcher, 2012). For the purposes of the current chapter, we wish to focus on overarching models of action based upon these principles before we go on to consider neuropsychiatric perturbations in light of these models.

Frameworks for Agency: The Comparator Model, Predictive Coding, and Active Inference

Influential current theories of motor control are based on the pioneering work of Todorov and Jordan, who argued that sensory signals are passed down the motor hierarchy as motor commands in order to achieve desired action goals (Todorov \& Jordan, 2002; Todorov, 2004). According to computational models adhering to this now well-established optimal control theory, this transformation and selective use of sensory signals to specify motor commands entails two types of models (Wolpert \& Kawato, 1998). First, an inverse model selects appropriate motor commands that would achieve a desired goal. As these motor commands are sent to the muscles, an efference copy of the same commands is sent to an internal predictive, or forward, model. This model's task is to estimate the likely sensory consequences of the motor command and thus the intended action. The sensory predictions of this forward model are used to optimize the estimated state of the motor plan required by the inverse model. Sensory feedback provides additional information about the executed movement, but such sensory transmission is relatively slow. The advantage of the joined action of the inverse and forward models is that it can bypass these sensory delays, allowing rapid adjustments and fluent movements toward a desired goal. More generally, the optimal control of action is thought to depend to a large extent on the coordination of inverse and forward models through a series of comparators, the results of the comparisons being used to correct errors, deviations, and other regulatory purposes.

These theories were designed to understand motor control and performance, rather than the subjective experience of action and its control. In fact, as mentioned, several components of such motor control schemes are considered to be unconscious. Nevertheless, such models have proven useful in guiding investigations into which aspects of such motor generation schemes are linked to the subjective feelings of action awareness and agency (for reviews, see Desmurget \& Sirigu, 2009; Frith et al., 2000). Thus, according to such perspectives, our SoA depends on the degree of congruency between the 
predicted and actual consequences of our movements, with this mismatch being (p.353) continuously monitored as an action unfolds. Moreover, and crucially, predictions made by forward models are used to filter sensory information and to attenuate the sensory effects of self- versus other-generated movements, thus generating a marker of agency (which, as mentioned, is signified by an absent or low mismatch). Similarly, action awareness is thought to rely mainly on forward signals and related comparisons, while actual sensory feedback may not be necessary to construct motor awareness, particularly when the desired goal is achieved and there are no unexpected delays (Fourneret \& Jeannerod, 1998; Sarrazin et al., 2008). If, however, the goal is not achieved, or there are large errors or delays in the process of execution, then a comparator detects the mismatch between the expected and actual sensory feedback and awareness is updated.

Predictive coding models form a general and, in neuroscience, increasingly influential class of models accounting for perception and action (see also Box 16.1). In essence, the predictive coding perspective represents a means of (p. 354) (p.355) estimating the likely cause of a given set of sense data based upon the data themselves as well as prior information. In the case of SoA and motor awareness, this amounts to the estimation of the probability that one was the agent of an action given the sensory and perceptual attributes of that action (for example, proprioception, sensorimotor feedback, external cues) and the prior probability that the action was executed and one was indeed the agent. Such a framework may, of course, exist at multiple levels arranged hierarchically. In the context of a model of brain function that posits the brain as seeking to minimize prediction error, active inference refers to the attempt to predict future movements through the representation of intentions (Adams et al., 2013; Friston et al., 2011).

\section{Box 16.1 A Computational Neuroscience Framework for Examining} Agency

The starting point of the free energy framework (Friston, 2005) is that the world is an uncertain place for self-organizing biological agents to survive. The signals that an organism may receive from the world may be caused by several, unknown causes. This inherent ambiguity of the world specifically threatens our need to occupy a limited repertoire of sensory states (e.g., humans need certain ranges in environmental temperature in order to survive). If, however, we cannot predict the causes of possible changes in the world (e.g., the "weather") with any certainty, we may find ourselves in surprising states for longer periods than those we could biologically sustain (e.g., in cold climates). We thus come up with a cheeky and yet no less ingenious solution. We base our predictions about our sensory states on 
unconscious inferences about their causes in the world (von Helmholtz, 1866). On the basis of limited or noisy information, our brain engages in some form of probabilistic representation of the causes of our future states in an uncertain world so that it maintains hypotheses ("generative models") of the hidden causes of sensory input. Theoretical neuroscientists use Bayesian theory to formalize this kind of inference and a number of other computational terms about probability distributions, such as "free energy," "uncertainty," and "surprise" that have the advantage of being formally (mathematically) defined. We have not addressed the issues of interest in mathematical ways here. Instead we have attempted to find faithful "psychological translations" for the mathematical definition of these concepts in order to examine clinical and experimental evidence on motor agency and awareness. Later in the chapter we outline the general ideas behind such a "psychologized" version of the free energy framework.

The central tenet of this framework is that the brain attempts to reduce the probability of being surprised by the world. It does this by deriving-from genes and experience-inferential, predictive models of possible causes of its sensory input. Errors in the accuracy of such representations have been conceptualized as free energy, on the basis of the formal definition of the latter-a quantity from informational theory that bounds (is greater than) the evidence for a model of data (Feynmann 1972; Hinton \& van Camp, 1993). The brain's data are sensory, and free energy bounds the negative logevidence (surprise) inherent in sensory data, given a model of how the data were caused. Furthermore, in agreement with the so-called predictive coding scheme (Rao \& Ballard, 1999), our brain is assumed to achieve the minimization of free energy by recurrent message passing among hierarchical level of cortical systems, so that various neural subsystems at different hierarchical levels minimize uncertainty about incoming information by structurally or functionally embodying a prediction (or a prior) and responding to errors (mismatches) in the accuracy of the prediction: so-called prediction errors. Such prediction errors are passed forward to drive the units in the level above that encode conditional expectations that optimize top-down predictions to explain away (reduce, inhibit) prediction error in the level below until conditional expectations are optimized. Such message passing is considered neurobiologically plausible on the basis of functional asymmetries in cortical hierarchies (see Mesulam, 2012), where forward connections (which convey prediction errors) are driving and backward connections have both driving and modulatory characteristics (thus modeling the nonlinear generation of sensory input). Given some mathematical assumptions, free energy can be thought of as the amount of prediction error in any given level of the system, including both exteroceptive and interoceptive (Seth et al., 2012) prediction errors. 
Minimizing free energy then corresponds to explaining away prediction errors following the principles of Bayes (Friston, 2010).

However, representing the world in constructive ways (perceptual inference) cannot take us far in terms of our ultimate goal, which is surviving in an uncertain world. Psychologically speaking, we may become better in predicting ("mentalizing") the changes in the environment that act to produce sensory impressions on us, but we cannot on this basis change the sensations themselves and hence ultimately their surprise. A highly innovative conceptual move in the free energy principle framework allows us to understand how we do just that. By acting upon the world, we can change its states and therefore "re-sample" the world to ensure that we satisfy our predictions about the sensory input we expect to receive. By selectively sampling the sensory inputs that we expect, we add accuracy to our predictions about sensory states. This view suggests that action is best understood as being elicited to fulfill prior expectations about proprioceptive sensations, not desired sensory states (as classic optimal motor control theory would suggest). Thus, action has an intimate relationship with perception, both being governed by the same master principle, namely reduction of prediction error. Action therefore becomes a means of adhering to the central principle of avoiding surprising states or minimizing prediction error. Thus action can reduce free energy by changing sensory input, while perception reduces free energy by changing predictions. According to the framework, our predictions (or priors) thus become a constantly updated, iterative, self-fulfilling prophecy that allows us to evade the inherent surprise of the world.

Finally, according to the framework, the organism needs to probabilistically infer two properties of the world: its states (content; mathematically this can be thought of as the center of a probability distribution), and the uncertainty (context; the dispersion of such distribution) about such states. Thus, optimal inference in both perception and action requires optimizing the precision (mathematically inverse amplitude or variance, and hence the inverse of uncertainty) of sensory signals (Feldman \& Friston, 2010; Friston et al., 2012a). Uncertainty is thought of as encoded mainly by synaptic gain that encodes the precision of random fluctuations about predicted states. It follows that neuromodulations of synaptic gain (such as dopamine and acetylcholine) do not signal (reward or pleasure) prediction errors about sensory data but the context in which such data were encountered. In other words, such neuromodulators report the salience of sensorimotor representations encoded by the activity of the synapses they modulate. This is important, especially in hierarchical schemes, where precision controls the relative influence of bottom-up prediction errors and top-down predictions. As regards exteroception, this processing of salience can be seen as attention in perceptual inference (Feldman \& Friston, 2010), and as 
affordance (latent action possibilities of cues in the environment) in active inference (Friston et al., 2012a). It has recently been proposed that optimizing the precision of internal body signals can be seen as increased interoceptive sensitivity and related feelings of arousal in perceptual inference and as increased seeking behaviours in active inference (Fotopoulou, 2014).

While it is beyond the scope of this chapter to deal with these models in detail, it is worth mentioning that the comparator and active inference models of SoA are different in certain fundamentals. While both speculate on the (p.356) importance of minimizing a mismatch or prediction error signal in controlling movement and generating SoA, the comparator model relates this to motor commands, while active inference focuses on proprioceptive predictions wherein a movement is enacted as a means of resolving a proprioceptive prediction error (emerging from the mismatch between the goal position [i.e., the intention] and the current position). Given that the movement would violate a prediction that the person is not moving (and therefore should militate against movement given the framework that actions are taken to minimize prediction error), it has been speculated (Brown et al., 2013) that the key occurrence that releases the desired movement is a reduction in the precision of sensory prediction error.

This reduced precision of sensory prediction error is the cause of sensory attenuation (rather than an absence of mismatch as posited by the comparator model) and thus, like the comparator model, the active inference model can account for the characteristic sensory illusion seen in a force-matching task (Brown et al., 2013), though they invoke quite different processes to explain this effect. Although we cannot cover this evidence in full here, it is worth mentioning that this model addresses a broader concept of sensory attenuation than the comparator model, including changes in the sensitivity of responses to external stimuli and not just the criteria of responses (see Brown et al., 2013, p. 3 for further discussion). The emergence of abnormal SoA in schizophrenia is likewise accounted for in quite different ways by the two models, but these precise differences are necessarily beyond the scope of this chapter. We will return to some of the fundamental difference in the sections on abnormalities of agency.

\section{Aberrant Agency and Motor Awareness}

As mentioned, sense of agency and motor awareness are not, even in health, infallibly accurate. Individuals may falsely claim to be the agents of actions or outcomes that they did not cause. Conversely, they may fail to attribute agency to actions that they did indeed cause. A celebrated example of the latter is the "table-turning" phenomenon of the nineteenth century. Here, the experience among groups of people was of a table moving under their hands, propelled, they believed, by members of the spirit world. This strong but erroneous SoA 
was, presumably, a consequence of prior beliefs (that spirits could and would move the table) acting on weak sensorimotor experiences. The latter would be especially vulnerable given that this occurred within a group such that the force exerted by any individual would be insufficient to move the table, while the experience of the table turning (as a consequence of the group effort) would be striking. In addition to a failure to experience agency even when one was (p. 357) indeed the author of an action, the converse is possible. Wegner and Wheatley have, as described above, shown how appropriately presented cues or primes can produce a feeling that one was the primary agent of an action that was, in fact, initiated by another (Wegner \& Wheatley, 1999). Given the objective imperfections of SoA as a marker for self- versus externally generated actions, it is unsurprising that a number of neuropsychiatric illnesses are associated with profound alterations in the experience of agency. Moreover, while recent studies in cognitive neuroscience have used several experimental "tricks" to systematically manipulate sensorimotor signals, to promote their integration, or to generate conflicts and illusions with a view to studying their role in SoA and motor awareness, these studies in healthy volunteers are by necessity constrained by the duration and setup of the experiment. To date, neuropsychiatric disorders that entail relatively long-lasting and biologically induced abnormalities in the subjective experience of agency and motor awareness represent an additional, indispensable window of insight into the neurocognitive mechanisms underlying such subjective feelings. Here we will attempt to apply the insights afforded by the aforementioned theoretical models of SoA and motor awareness in the consideration of three prototypical disorders of agency and motor awareness, namely (1) delusions of control in psychosis; (2) anosognosia for hemipleagia following stroke; (3) anarchic hand syndrome following brain damage. We believe such consideration may enhance the understanding of the pathogenesis of these disorders, as well as the mechanisms underlying the SoA and motor awareness more generally.

\section{Agency and Psychosis: Delusions of Control}

Psychosis refers to a loss of contact with reality. It is characterized by abnormal perceptions (specifically, perceptions in the absence of objectively quantifiable causal stimuli) and abnormal (bizarre, apparently irrational, persistent) beliefs, referred to as hallucinations and delusions, respectively. Psychosis can occur across an array of psychiatric and neurological disorders and is perhaps most traditionally associated with schizophrenia. An intriguing and compelling point that has been frequently made with respect to psychosis is that a core abnormality appears to lie in misattribution-specifically, misattribution of selfgenerated thoughts or acts to the external world. Thus, for example, an auditory hallucination in the form of a voice has been conceived as a misattribution of internal speech to an external agent and, hence, its perception as some external agent speaking. A particularly interesting feature of schizophrenia in this regard is the passivity phenomenon wherein the sufferer perceives him- or herself to be 
the passive recipient of actions, thoughts, or emotions. Such experiences are highly complex but do seem to entail, at least (p.358) in part, a disturbed SoA. One striking experience is the delusion of motor control, wherein someone's motor actions feel as though they have been externally caused. Indeed, they may be attributed to some unseen persecutor who, in some mysterious way, has attained control over the sufferer's body. Notably, this may occur in the context of movements that were preceded by an intention to move and that fulfilled the object of this intention.

Aside from the fact that they pose something of a challenge to any simple distinction between hallucinations and delusions (seeming simultaneously to entail both an abnormal perception and an abnormal belief), delusions of motor control represent a profound explanatory challenge. In the context of intended movements, successfully carried out, what might account for such an unusual experience? One compelling response to this challenge emerges from the comparator model of motor control alluded to earlier. To recap, this model suggests that an intention toward action engenders, via an inverse model, a series of planned movements that leads, via a forward model, to a predicted state (the new set of sensory and proprioceptive input that should obtain following the successful completion of those movements). Critically, there is a comparison between these two states (the predicted and the actual state), which of course is necessary to modulating movement in order to achieve ultimately the intended goal. A small or absent discrepancy between the two states signifies a successful movement, but it is also a hallmark of agency: after all, a large discrepancy, signaling a surprising state following a movement, would be a sign that the movement was neither intended nor under control. This simple model makes a prediction elegantly tested and confirmed by Shergill and colleagues: specifically, actions for which one is the agent will have predictable sensory consequences, which can then be canceled or ignored; that is, selfgenerated actions will be associated with dampened sensory consequences. They tested this using a "force-matching" task in which participants used selfgenerated force to match a force that had just been applied to them externally. Results suggest that self-generated force is indeed experienced as less than externally generated force (Shergill et al., 2003).

The comparator model may be simply extended to explain delusions of motor control. The failure to construct a forward model leads to erroneous predictions of the consequences of one's actions, which are therefore not dampened and, hence, the action has the hallmarks not of self- but of external generation. The evidence for this comes from observations that people with schizophrenia show results on the force-matching task suggestive of failed sensory dampening and, indeed, find their own actions more intense than do control subjects (Shergill et al., 2005). 
As discussed, an alternative perspective is offered by an Active Inference model in which it is a prediction error generated by a mismatch between the (p.359) current state and the predicted future state that forms the drive to action (mediated by reflex arcs). Importantly, in such models, the instantiation of an action necessarily entails an alteration (attenuation) in the precision, and hence weighting, of sensory evidence during the movement. This attenuation prevents a sensory prediction error from stifling the movement and, furthermore, can be invoked to explain (see Brown et al., 2013) the sensory attenuation illusion as well as the absence of such attenuation in noted in schizophrenia.

\section{Anosognosia for Hemiplegia}

Anosognosia for hemiplegia (AHP) is the apparent inability to acknowledge one's contralesional paralysis following stroke-induced perisylvian lesions, typically to the right hemisphere (though there are exceptions; Cocchini et al., 2009). This counterintuitive but prototypical neurological disorder of motor unawareness typically lasts from days to weeks, but even at the acute stages of the illness patients may show strong delusional beliefs and corresponding emotional attitudes toward the paralyzed body parts. In some patients such beliefs seem to be linked to their claims that their limbs have moved even upon demonstration of the opposite (illusory movements; Feinberg et al., 2000; Fotopoulou et al., 2008), while others may admit their on-line failures, but fail to update their "offline" body awareness, or acknowledge the functional consequences of their paralysis (Carruthers, 2008; Marcel et al., 2004). Interestingly, despite such illusions and delusions, some of these patients may show implicit awareness of their deficits in verbal (Fotopoulou et al., 2010) or behavioral tasks (Cocchini et al., 2010; Moro et al., 2011; Nardrone et al., 2007), and they may be more aware of their motor failures when taking a third- as opposed to a first-person perspective (Fotopoulou et al., 2009, 2011; Marcel et al., 2004).

Initially, AHP was explained as the secondary consequence of one or more of the concomitant sensorimotor and cognitive impairments that frequently accompanied it, such as primary sensorimotor deficits, generalized cognitive impairment, or neglect (for reviews, see Jehkonen et al., 2006; Orfei et al., 2007). Several studies, however, have revealed double dissociations between AHP and such impairments (e.g. Bisiach et al., 1986; Marcel et al., 2004), suggesting that they are not necessary for AHP to occur, although they could act as contributing factors.

Influenced by the aforementioned "comparator" models, some investigators have argued that AHP is not the secondary consequence of deficits in other domains, but rather the primary outcome of abnormalities in encapsulated and modular mechanisms of anticipatory motor awareness (Berti et al., 2005; Frith et al., 2000; Heilman et al., 1998). Not all of these perspectives, however, (p.360) propose the same component of the comparator model as responsible for AHP. Heilman and colleagues (Heilman et al., 1998) have proposed that AHP arises 
from a failure to form motor intentions, resulting in the forward model not priming the comparator to expect movement, and hence patients never "discover" that they cannot move. Frith and colleagues (2000) alternatively proposed that although patients with AHP are able to predict the expected sensory consequences of intended movements, they fail to register the discrepancy between predicted and actual sensory feedback because of visuospatial neglect or other sensory deficits. Berti and colleagues (Berti et al., 2005) follow Frith and colleagues in proposing that patients are unaware of the discrepancy between intended and actual movement; however, they suggest that this failure to detect discrepancies is the result of damage directly to the comparator. The latter two hypotheses are capable of accounting for both the negative (unawareness of motor failures) and the positive (the illusory awareness of having moved) signs of AHP, while the theory of Heilman and colleagues addresses only the negative signs.

Empirical findings thus far have supported mainly the explanation put forward by Berti and colleagues (see Jenkinson \& Fotopoulou, 2010, for review). A lesionmapping study (Berti et al., 2005) revealed that the brain areas involved in monitoring the correspondence between motor commands and sensory feedback (i.e., Brodmann premotor areas 6 and 44 and the insular cortex) are selectively damaged in patients with AHP, while areas typically responsible for motor planning (e.g., supplementary motor cortex) are intact in these patients. Moreover, there is physiological (Berti et al., 2007; Hildebrandt \& Zieger, 1995; but see Gold et al., 1994) and behavioral (Garbarini et al., 2012; Jenkinson, Edelstyn, \& Ellis, 2009) evidence for the presence of intact motor intentions in AHP. A further study showed for the first time the direct relation between motor intention and awareness (Fotopoulou et al., 2008). Specifically, patients' illusory awareness of movement, and related feelings and judgements of agency (was it you or someone else who performed the action?) reflected an abnormal, selective dominance of motor intentions over visual feedback about the actual effects of movement (elicited by a realistic rubber hand that patients assumed was their own). Further, this effect could not be explained by neglect.

Despite the clear value of the "feed-forward" hypotheses, it has become apparent that a strictly modular, motor explanation is not sufficient to account for all the manifestations of AHP (e.g., Fotopoulou et al., 2010; Orfei et al., 2009; Vocat et al., 2010). Feed-forward theories are valuable in explaining the illusion of moving, but AHP patients do not simply claim that they have the phenomenal experience of moving. Instead, they ignore the wealth of contrary evidence and medical signs indicating that they are paralyzed (e.g., their medical results, disabilities, occasional accidents, and others' feedback) and they (p.361) adhere to the delusional belief that they have functional limbs, showing corresponding emotional attitudes. The explanation of such beliefs and attitudes requires the postulation of additional dysfunctions that prevent sensorimotor 
and other failures from being re-represented at a higher level of cognitive and emotional self-representation, one that is beyond the sensorimotor domain.

This is the point at which predictive coding schemes become useful. Unlike optimal motor control theory, such models can envision a mismatch between prediction and experience in various levels of the neurocognitive hierarchy and in relation to several cognitive and emotional domains. For example, they can explain the motor illusions of patients who claim they have moved their arms as planned, even upon demonstration of the contrary (Fotopoulou et al., 2008), but they can also explain the more general, obstinate adherence of other patients to their premorbid everyday habits ("of course, I can walk") despite implicit knowledge of their paralysis (Fotopoulou et al., 2010).

Specifically, aberrant perceptual inference (suboptimal synaptic activity; Friston, 2010) can be caused by deficits that lead to weak, absent, or unreliable prediction errors, and hence lead patients to base their inference on premorbid, non-updated predictions about their motor abilities (see also Fotopoulou, 2012, 2013). Such deficits may well occur in relation to exteroceptive signals about the left side of the body as represented in the connections of right hemisphere subcortical areas (e.g., the thalamus), or re-represented and organized in cortical functional networks of the right hemisphere (Berti et al., 2005;

Fotopoulou et al., 2010; Moro et al., 2011; Vocat et al., 2010). However, as aforementioned, these deficits are not necessary to cause AHP. They can readily explain illusions of moving (like the comparator model does), but not the more general delusion of being able to move. Interestingly, recent lesion-mapping studies have highlighted that areas such as the insula, limbic structures, and subcortical white matter connections may be selectively associated with AHP (Fotopoulou et al., 2010; Karnath et al., 2005; Moro et al., 2011; Vocat et al., 2010). Such areas are linked with interoception and motivation and are specifically implicated in bodily salience and interoceptive awareness (Craig, 2003; Critchley et al., 2004). Thus, we propose that weak or imprecise (see also below) interoceptive signals about the current state of the body may lead to persistent adherence to past expectations of how the affected body parts should feel leading to the ensuing aberrant beliefs. Moreover, given the position of such priors in the neurocognitive hierarchy, such faulty inference may also "explain away" contrary exteroceptive signals in some patients. To use the words of one anosognosic patient who also denied the ownership of his paralyzed limbs, "But my eyes and my feelings don't agree, and I must believe my feelings. I know they [left arm and leg] look like mine, but I can feel they are not, and I can't believe my eyes" (C. W. Olsen, 1937, cited in Feinberg, 1997).

(p.362) It is worth noting that the principle of Free Energy minimization may enhance the understanding of AHP and motor awareness in at least one additional way. First, the recently identified lesions in frontostriatal circuits (Fotopoulou et al., 2010; Moro et al., 2011; Venneri \& Shanks, 2004; Vocat et al., 
2010) may have a modulatory role in AHP, leading to dopamine depletion and a more general difficulty in optimizing the precision (uncertainty) of prediction errors (Friston et al., 2012), affecting their salience and ultimately both shortand long-term learning (suboptimal synaptic gain and plasticity; Friston, 2010). Indeed, the functional role of the basal ganglia and particularly the striatum has been linked with prediction error-driven learning (O’Doherty et al., 2003) as well as the aberrant salience theories of psychosis (Gray et al., 1991; Kapur, 2003), which as we saw have direct implications for the formation of delusions about one's agency in psychosis. In AHP such deficits can be linked with both specific instances of aberrant motor monitoring in functionally specialized systems (Berti et al., 2005), or more generally in global error monitoring (Davies et al., 2005; Venneri \& Shanks, 2004; Vocat et al., 2012), mental flexibility (Levine et al., 1991), and "surprise detection" (Ramachandran, 1995) deficits. Indeed, a recent study showed that AHP patients had the tendency to "jump to conclusions" on the basis of limited and rather vague information and then to subsequently get stuck to their former "false" beliefs instead of modifying them based on novel, arguably more salient information (Vocat et al., 2012).

\section{Anarchic Hand}

Another counterintuitive neuropsychiatric syndrome challenges the commonsense notion that our actions are caused by a central, unitary will (Libet, 1983). Contrary to anosognosic patients who may erroneously feel that they have executed intended movements with their paralyzed limbs, patients with "anarchic hand sign" (AHS) have usually distressing experience of one of their arms acting without being guided by the patient's conscious will (for a review, see Fisher, 2000). The hand is indeed executing the movements that patients report, but it appears to act entirely on its own accord, grabbing objects that the patient had no known intention of wanting to grab, or not releasing objects the patient wishes to release. Interestingly, the actions that the affected arm performs, although not intended, are nevertheless purposeful in themselves (e.g., open a door, take off clothes) and are typically completed successfully. In some patients the affected arm may even hinder purposeful actions of the other arm (intermanual conflict, or diagonistic dyspraxia). The patients do not deny the ownership of the hand (see also below), nor the fact that their own (p.363) body is actually executing the actions. However, they experience the hand as "having a will of its own," or of being controlled by external agents.

There appears to be some long-standing and persevering taxonomical and nosological confusion in the neurological literature of this syndrome. For starters, the term "alien hand" syndrome or sign is frequently used to refer not only to the presence of involuntary, uncontrolled movements in one's limbs but also to feelings of non-belonging (lack of ownership) for the affected limb in the absence or presence of such involuntary movements. Thus, Della Sala and colleagues (e.g. Marchetti \& Della Sala, 1998) proposed restricting the term "alien hand" to conditions involving the feeling of non-belonging of a hand (lack 
of the sense of ownership for the arm) and introduced "anarchic hand" to refer to conditions where subjects perform involuntary movements with their hand but acknowledge the ownership of the same hand. We follow this distinction in this chapter and below we attempt to explain the neuropsychological mechanisms that are responsible for the AHS specifically.

The AHS can be caused by stroke, midline tumors, corticobasal degeneration, or callosotomy for epilepsy, and it can be as variable as it is rare. In its most typical and dramatic form, AHS arises following lesions to the medial frontal lobes and the corpus callosum. However, the syndrome can also be observed in association with subcortical (mainly to the thalamus and basal ganglia) and posterior (parietal cortex and posterior corpus callosum) lesions. Various related distinctions have been proposed, such as anterior versus posterior AHS, or frontal versus callosal AHS, but their validity and relation to laterality remains unclear (see Kikkert et al., 2006, for review). It seems more appropriate to label the syndrome according to the affected hemisphere in each case, as well as to specify which of the common behavioral symptoms are present in each case; for example, Aboitiz et al. (2003) suggest that apart from the spontaneous, involuntary movements of the affected hand, the general syndrome could comprise (1) diagnostic dyspraxia (intermanual conflict), (2) alien hand (disownership of the hand, see also above), (3) supernumery hands (the experience of additional arms belonging to the patient), and (4) agonistic dyspraxia (involuntary movements of the affected arm with temporary inhibition of the other intact arm following bimanual instructions).

Experimental and functional neuroimaging studies in AHS are rare, and comparisons between them are hampered by the above taxonomical issues. However, most studies seem to have focused on two functional abnormalities of the "anarchic" behavior. First, in patients with AHS, simple observation of certain objects with strong motor affordances (Gibson, 1979) might be sufficient to elicit the associated motor plan for interacting with that object and lead to the corresponding action, even when such action conflicts with other motor goals (e.g., Humphreys \& Riddoch, 2000; Riddoch et al., 1998). Such (p.364) affordances' effects have been long shown in healthy individuals, for example in paradigms where visual objects and shapes can prime specific actions. The existence of such effects makes sense if one thinks that, in order to act successfully upon the world, we need to have readily available information about the position and shape of objects in relation to both our potentially moving body and the environment. This means that we need to be able to represent the relation between the two in various frames of reference and translate between them, as for example when we need to use visual cues, coded in retinotopic coordinates, to make a limb movement (coded in body-centered coordinates) toward an object (Jeannerod et al., 1995). There is well-established evidence that the parietal cortex supports such transformation abilities and maintains representations of the appropriate movement trajectories needed to reach and 
grasp objects of previously learned affordances. However, in patients with AHP the effects of visual affordances on action appear to be stronger in the anarchic hand relative to the unaffected hand (McBride et al., 2013), and such effects seem to actually lead to involuntary actions.

The latter observation brings us to the second reported abnormality in AHS. It has been proposed that these patients lack the ability to voluntarily suppress or inhibit the actions primed by the perceptual processing of objects (e.g., Biran et al., 2006; Giovannetti et al., 2005; Schaefer et al., 2010). However, more recently there is growing evidence in healthy volunteers that unconsciously primed responses (for reviews, see Eimer and Schlaghecken, 2003; McBride et al., 2012), or responses afforded by the properties of objects themselves (e.g., Vainio, 2009; Vainio \& Mustonen, 2011), can also be automatically and unconsciously inhibited. Moreover, such inhibition seems to be causally linked to the functional role of medial frontal cortex regions. For example, while the use of a recently developed masked-prime task (Eimer \& Schlaghecken, 2003) leads to an automatic inhibition of unwanted motor plans (activated unconsciously by condition-action associations) in healthy individuals, a recent study administered this paradigm to two patients with highly selective lesions to the supplementary eye field and motor area and found that such inhibition does not take place for hand and eye movements, respectively (Sumner et al., 2007). Consistently, the same priming paradigm has been used in patients with AHS (where damage to such medial frontal cortex areas is common but may not be as specific) to show that such automatic inhibition no longer takes place, and instead perceptually processed affordances are automatically translated into the execution of the corresponding actions (McBride et al., 2013).

From the perspective of the comparator model, the AHS has been explained as an inappropriate activation of the parietal cortex areas responsible for the perceptual and visuomotor processing of objects due to damage to the supplementary motor cortex (SMA), which is normally responsible for action selection and (p.365) corresponding inhibitory functions (Frith et al., 2000; see also above). In terms of the relation of such damage to the motor control model, it is assumed that the current motor intentions of the patient are no longer able to inhibit the visuomotor effects afforded by the environment and thus patients execute whatever action is afforded by the object in front of them. Furthermore, according to the model, our motor awareness derives from the various comparisons between the desired, predicted, and actual state of the body and, as aforementioned, we normally have a limited awareness of our motor commands themselves and the way immediate sensory information (affordances) are used to fine-tune such commands (the ways in which the controller selects and corrects the precise commands required for an action). The areas that underlie the desired, predicted, and actual states of the body are considered intact in 
patients with AHS and hence these patients are aware of the fact that their arm executes actions that differ from their own, conscious intentions.

As described above, from the perspective of predictive coding and active inference, descending signals from higher motor areas to the periphery are conceptualized as proprioceptive predictions (not motor commands) and the corresponding ascending signals (proprioceptive prediction errors) are understood to fulfill such priors by leading to action and correcting predictions at lower levels of the hierarchy, rather than passing up to higher areas and changing the intended actions. Thus, in the context of this model, the fact that patients with AHS do execute successful movements with their affected arm and are aware of having executed them suggests that at some level in the sensorimotor hierarchy proprioceptive predictions were formed and fulfilled. Moreover, unlike in the case of delusions of alien control (see above), one may expect that the precision of sensory prediction errors is attenuated to some degree as patients do not attribute their actions to a different agent. It seems, however, that the formed and fulfilled proprioceptive predictions have been generated in parietal or subcortical sensorimotor areas as a result of external affordances and without the top-down involvement of certain damaged, or disconnected (by callosal damage) higher-order motor areas such as the SMA. Thus, we speculate that large prediction errors arise at high levels of the hierarchy because of the discrepancy between the sensory predictions of spared higher order sensorimotor motor areas and the prediction errors (posterior beliefs about the executed movements) conveyed by lower motor areas.

Conclusion

Though ubiquitous and compelling, our sense of being the agent of our actions is complex, mutable, and unreliable. Moreover, the scientific frameworks and means of measurement used to research this subjective experience are necessarily approximate and, at times, questionable. Against this background we (p.366) have reviewed two types of model used as frameworks for understanding SoA and have highlighted key areas in which they differ. Importantly, we argue that neuropsychiatric disorders may provide very useful windows onto agency. Interestingly, with some exceptions, such disorders have not, yet, been the subject of comprehensive and systematic studies capitalizing on technological advances in neuroscience. It seems likely that the increasing sophistication of computational theories of motor function may provide a useful platform in this regard. It is also worth noting in closing that such models, as they become more general and depart from a strictly motor emphasis (for example, moving from comparator models to predictive coding and active inference models), may offer ways of providing a more comprehensive understanding of the whole range of features accompanying such syndromes.

\section{References}


Bibliography references:

Aboitiz, F., Carasco, X., Schröter, C., Zaidel, D., Zaidel, E., \& Lavados, M. (2003). The alien hand syndrome: classification of form reported and discussion of a new condition. Neurological Sciences, 24, 252-257.

Adams, R. A., Shipp, S., \& Friston, K. J. (2013). Predictions not commands: active inference in the motor system. Brain Structure \& Function, 218(3), 611-643.

Berti, A., Bottini, G., Gandola, M., Pia, L., Smania, N., Stracciari, A., Castiglioni, I., Vallar, G., Paulesu, E. (2005). Shared cortical anatomy for motor awareness and motor control. Science, 309, 488-491.

Biran, I., Giovannetti, T., Buxbaum, L., and Chatterjee, A. (2006) The alien hand syndrome: what makes the alien hand alien? Cognitive Neuropsychology, 23(4), 563-582.

Bisiach, E., Vallar, G., Perani, D., Papagno, C., Berti, A. (1986). Unawareness of disease following lesions of the right hemisphere: anosognosia for hemiplegia and anosognosia for hemianopia. Neuropsychologia, 24, 471-482.

Brown, H., Adams, R. A., Parees, I., Edwards, M., Friston, K. (2013). Active inference, sensory attenuation and illusions. Cognitive Processing, 14, 411-427.

Carruthers, G. (2008). Types of body representation and the sense of embodiment. Consciousness and Cognition, 17, 1302-1316.

Cocchini, G., Beschin, N., Cameron, A., Fotopoulou, A., \& Della Sala, S. (2009). Anosognosia for motor impairment following left brain damage.

Neuropsychology, 23(2), 223-230.

Cocchini, G., Beschin, N., Fotopoulou, A., \& Della Sala, S. (2010). Explicit and implicit anosognosia for upper limb motor impairment. Neuropsychologia, 48(5), 1489-1494.

Craig, A. D. (2003). Interoception: the sense of the physiological condition of the body. Current Opinion in Neurobiology, 13, 500-505. doi: 10.1016/

S0959-4388(03)00090-4

Critchley, H. D., Wiens, S., Rotshtein, P., Öhman, A., \& Dolan, R. D. (2004).

Neural systems supporting interoceptive awareness. Nature Neuroscience, 7 , 189-195.

Crosson, C., Barco, P., Velozo, C., Bolesta, M., Cooper, P., Werts, D., et al. (1989). Awareness and compensation in postacute head injury rehabilitation. Journal of Head Trauma Rehabilitation, 4, 46-54. (p.367) 
Davies, M., Davies, A. A., \& Coltheart, M. (2005). Anosognosia and the twofactor theory of delusions. Mind and Language, 20, 209-236.

Desmurget, M., \& Sirigu, A. (2009) A parietal-premotor network for movement intention and motor awareness. Trends in Cognitive Sciences, 13, 411-419.

Eimer, M., \& Schlaghecken, F. Response facilitation and inhibition in subliminal priming. Biological Psychology, 64(1e2): 7e26, 2003.

Ernst, M. O., \& Bulthoff, H. H. (2004). Merging the senses into a robust percept. Trends in cognitive sciences, 8(4), 162-169

Feinberg T. E. (1997). Anosognosia and confabulation, In: Feinberg T.E., Farah M. editors. Behavioral neurology and neuropsychology. New York: McGraw-Hill, 1997: 369-390.

Feinberg, T. E., Roane, D. M., \& Ali, J. (2000). Illusory limb movements for anosognosia for hemiplegia. Journal of Neurology, Neurosurgery and Psychiatry, $68,511-513$.

Feynman, R. P. (1972). Statistical Mechanics. Reading: Benjamin.

Fisher, C. M. (2000). Alien hand phenomena: a review with the addition of six personal cases. The Canadian Journal of Neurological Sciences, 27(3): 192-203.

Fotopoulou, A. (2014). Time to get rid of the "modular" in neuropsychology: a unified theory of anosognosia as aberrant predictive coding. Journal of Neuropsychology, Mar;8(1), 1-19.

Fotopoulou, A. (2014) Beyond the reward principle: consciousness as precision seeking. Neuropsychoanalysis, 15(1), 33-38.

Fotopoulou, A. (2012). Illusions and delusions in anosognosia for hemiplegia: from motor predictions to prior beliefs. Commentary. Brain, 135, 1344-1347.

Fotopoulou, A., Tsakiris, M., Haggard, P., Rudd, A., \& Kopelman M. (2008). The role of motor intention in motor awareness: an experimental study on anosognosia for hemiplegia. Brain, 131, 3432-3442.

Fotopoulou, A., Jenkinson, P. M., Tsakiris, M., Haggard, P., Rudd, A., \& Kopelman, M. D. (2011). Mirror-view reverses somatoparaphrenia: dissociation between first- and third-person perspectives on the body. Neuropsychologia, 49(14), 3946-3955.

Fotopoulou, A., Pernigo, S., Maeda, R., Rudd, A., \& Kopelman, M. A. (2010). Implicit awareness in anosognosia for hemiplegia: unconscious interference without conscious re-representation. Brain, 133(Pt 12), 3564-3577. 
Fotopoulou, A., Rudd, A., Holmes, P., \& Kopelman, M. (2009). Self-observation reinstates motor awareness in anosognosia for hemiplegia. Neuropsychologia, 47(5), 1256-1260.

Fourneret, P., \& Jeannerod, M. (1998). Limited conscious monitoring of motor performance in normal subjects. Neuropsychologia, 36, 1133-1140.

Friston, K. (2005). A theory of cortical responses. Philosophical transactions of the Royal Society B: Biological sciences, 360(1456): 815-836.

Friston, K. (2010). The free-energy principle: a unified brain theory. Nature Reviews: Neuroscience, 11, 127-138.

Friston, K. J., Shiner, T., FitzGerald, T., Galea, J. M., Adams, R., Brown, H., Dolan, R. J., Moran, R., Stephan, K. E., \& Bestmannm, S. (2012). Dopamine, affordance and active inference. PLoS Computational Biology, 8(1), e1002327.

Frith, C. D., Blakemore, S. J., \& Wolpert, D. M. (2000) Abnormalities in the awareness and control of action. Philosophical Transactions of the Royal Society of London, Series B. Biological Sciences, 355, 1771-1788. (p.368)

Gallagher, S. (2000). Philosophical concepts of the self: implications for cognitive sciences. Trends in Cognitive Sciences, 4, 14-21.

Garbarini, F., Rabuffetti, M., Piedimonte, A., Pia, L., Ferrarin, M., Frassinetti, F., Gindri, P., Cantagallo, A., Driver, J., \& Berti, A. (2012). "Moving” a paralysed hand: bimanual coupling effect in patients with anosognosia for hemiplegia. Brain, 135(Pt 5), 1486-1497.

Gibson, J. J. (1979). The ecological approach to visual perception. Boston: Houghton Mifflin.

Giovannetti, T., Buxbaum, L. J., Biran, I., \& Chatterjee, A. (2005) Reduced endogenous control in alien hand syndrome: evidence from naturalistic action. Neuropsychologia, 43(1), 75e88.

Gold, M., Adair, J. C., Jacobs, D. H., \& Heilman, K. M (1994). Anosognosia for hemiplegia: an electrophysiologic investigation of the feed-forward hypothesis. Neurology, 44, 1804-1808.

Gray, J. A., Feldon, J., Rawlins, J. N. P., Hemsley, D., \& Smith, A. D. (1991). The neuropsychology of schizophrenia. Behavioral and Brain Sciences, 14, 1-84.

Heilman, K. M., Barret, A. M., \& Adair, J. C. (1998). Possible mechanisms of anosognosia: a defect in self-awareness. Philosophical Transactions of the Royal Society of London, Series B. Biological Sciences, 353, 1903-1909. 
Hildebrandt, H., \& Zieger, A. (1995). Unconscious activation of motor responses in a hemiplegic patient with anosognosia and neglect. European Archives of Psychiatry and Clinical Neuroscience, 246, 53-59.

Hinton, G. E., \& van Camp, D. (1993). Keeping neural networks simple by minimising the description length of weights. In: COLT '93 Proceedings of the Sixth Annual Conference on Computational Learning Theory. New York: Association of Computing Machinery, 5-13.

Humphreys, G. W., \& Riddoch, M. J. (2000). One more cup of coffee for the road: object-action assemblies, response blocking and response capture after frontal lobe damage. Experimental Brain Research, 133(1): 81-93.

Jehkonen, M., Laihosalo, M., \& Kettunen, J. (2006). Anosognosia after stroke: assessment, occurrence, subtypes and impact on functional outcome reviewed. Acta Neurologica Scandinavica, 114, 293-306.

Jenkinson, P., \& Fotopoulou, A. (2010). Motor awareness in anosognosia for hemiplegia: experiments at last. Experimental Brain Research, 204(3), 295-304.

Jenkinson, P. M., Edelstyn, N. M., Drakeford, J. L., \& Ellis, S. J. (2009). Reality monitoring in anosognosia for hemiplegia. Consciousness and Cognition, 18, 458-470.

Jeannerod, M., Arbib, M. A., Rizzolatti, G., \& Sakata, H. (1995). Grasping objects: the cortical mechanisms of visuomotor transformation. Trends in Neurosciences, 18, 314-320.

Kapur, S. (2003). Psychosis as a state of aberrant salience: a framework linking biology, phenomenology, and pharmacology in schizophrenia. American Journal of Psychiatry, 160, 13-23.

Karnath, H. O., Baier, B., \& Nägele, T. (2005). Awareness of the functioning of one's own limbs mediated by the insular cortex? Journal of Neuroscience, 3 , 25(31), 7134-7138.

Kikkert, M. A., Ribbers, G. M., \& Koudstaal, P. J. (2006). Alien hand syndrome in stroke: a report of 2 cases and review of the literature. Archives of Physical Medicine and Rehabilitation, 87, 728-732. (p.369)

Levine, D. N., Calvanio, R., \& Rinn, W. E. (1991). The pathogenesis of anosognosia for hemiplegia. Neurology, 41, 1770-1781.

Libet, B., Gleason, C. A., Wright, E. W., \& Pearl, D. K. (1983). Time of conscious intention to act in relation to onset of cerebral activity (readiness potential): the unconscious initiation of a freely voluntary act. Brain, 106, 623-642. 
Marcel, A. J., Tegner, R., \& Nimmo-Smith, I. (2004). Anosognosia for plegia: specificity, extension, partiality, and disunity of bodily unawareness. Cortex, 40, 19-40.

Marchetti, C., \& Della Salla, S. (1998) Disentangling the alien and anarchic hand. Cognitive Neuropsychiatry, 3, 191-208.

McBride, J., Sumner, P., Jackson, S. R., Bajaj, N., \& Husain, M. (2013).

Exaggerated object affordance and absent automatic inhibition in alien hand syndrome. Cortex, 49(8), 2040-2054.

McBride, J., Boy, F., Husain, M., \& Sumner, P. (2012). Automatic motor activation in the executive control of action. Frontiers in Human Neuroscience, 6, 82.

Mesulam M. (2012). The evolving landscape of human cortical connectivity: facts and inferences. Neuroimage, 62(4), 2182-2189.

Moore, J. W., \& Fletcher, P. C. (2012) Sense of agency in health and disease: a review of cue integration approaches. Consciousness and Cognition, 21(1), 5968.

Moore, J. W., Middleton, D., Haggard, P., \& Fletcher, P. C. (2012) Exploring implicit and explicit aspects of sense of agency. Consciousness and Cognition, 21(4), 1748-1753.

Moore, J. W., Teufel, C., Subramaniam, N., Davis, G., \& Fletcher, P. C. (2013). Attribution of intentional causation influences the perception of observed movements: behavioral evidence and neural correlates. Frontiers in psychology, 4.

Moore, J. W., Wegner, D. M., \& Haggard, P. (2009). Modulating the sense of agency with external cues. Consciousness and Cognition, 18(4), 1056-1064.

Moro, V., Pernigo, S., Zapparoli, P., Cordioli, Z., \& Aglioti, S. M. (2011). Phenomenology and neural correlates of implicit and emergent motor awareness in patients with anosognosia for hemiplegia. Behavioural Brain Research, 225(1), 259-269.

Nardone, I. B., Ward, R., Fotopoulou, A., \& Turnbull, O. E. (2007) Attention and emotion in anosognosia: evidence of implicit awareness. Neurocase, 13, 438445 .

O’Doherty, J. P., Dayan, P., Friston, K., Critchley, H., \& Dolan, R. J. (2003)

Temporal difference models and reward-related learning in the human brain.

Neuron, 38(2), 329-337. 
Orfei, M. D., Robinson, R. G., Prigatano, G. P., Starkstein, S., Rüsch, N., Bria, P., Caltagirone, C., \& Spalletta, G. (2007). Anosognosia for hemiplegia after stroke is a multifaceted phenomenon: a systematic review of the literature. Brain, 130(Pt 12), 3075-3090.

Orfei, M. D., Caltagirone, M., \& Spalletta, G. (2009). The evaluation of anosognosia in stroke patients. Cerebrovascular Disease, 27, 280-289.

Pacherie, E. (2007). The anarchic hand syndrome and utilization behavior: a window onto agentive self-awareness. Functional Neurology, 22(4) 211-217.

Ramachandran, V. S. (1995). Anosognosia in pariental lobe syndrome.

Consciousness and Cognition, 4, 22-51.

Rao, R. P., \& Ballard, D. H. (1999). Predictive coding in the visual cortex: A functional interpretation of some extra-classical receptive field effects. Nature Neuroscience, 2, 79-87.

Riddoch, M. J., Edwards, M. G, Humphreys, G. W., West, R., \& Heafield, T. (1998). Visual affordance direct action: neuropsychological evidence from manual interference. Cognitive Neuropsychology, 15(6/7/8): 645e-683. (p.370)

Sarrazin, J. C., Cleeremans, A., \& Haggard, P. (2008) How do we know what we are doing? Time, intention and awareness of action. Consciousness and Cognition, 17(3), 602-615.

Schaefer, M., Heinze, H. J., \& Galazky, I. (2010). Alien hand syndrome: neural correlates of movements without conscious will. PLoS One, 5(12), e15010.

Schacter, D. L. (1990). Toward a cognitive neuropsychology of awareness: implicit knowledge and anosognosia. Journal of Clinical and Experimental Neuropsychology, 12, 155-178.

Searle, J. R. (1983). Intentionality: an essay in the philosophy of mind. Cambridge: Cambridge University Press.

Seth, A. K., Suzuki, K., \& Critchley, H. D. (2012). An interoceptive predictive coding model of conscious presence. Frontiers in Psychology, 2(395), 1-16.

Shergill, S. S., Bays, P. M., Frith, C. D., \& Wolpert, D. M. (2003). Two eyes for an eye: the neuroscience of force escalation. Science, 301(5630), 187.

Shergill, S. S., Samson, G., Bays, P. M., Frith, C. D., \& Wolpert, D. M. (2005) Evidence for sensory prediction deficits in schizophrenia. American Journal of Psychiatry, 162(12), 2384-2386. 
Sumner, P., Nachev, P., Morris, P., Peters, A. M., Jackson, S. R., Kennard, C., \& Husain, M. (2007). Human medial frontal cortex mediates unconscious inhibition of voluntary action. Neuron, 54(5), 697-711.

Synofzik, M., Vosgerau, G., \& Newen, A. (2008). Beyond the comparator model: a multifactorial two-step account of agency. Conscious and Cognition, 17(1), 219239.

Todorov, E. (2004). Optimality principles in sensorimotor control. Nature Neuroscience, 7(9), 907-915.

Todorov, E., \& Jordan, M. I. (2002). Optimal feedback control as a theory of motor coordination. Nature Neuroscience, 5(11), 1226-1235.

Tsakiris, M., \& Fotopoulou, A. (2008). Is my body the sum of online and offline body representations? Consciousness and Cognition, 17(4), 1317-1320.

Vainio, L. (2009). Interrupted object-based updating of reach program leads to a negative compatibility effect. Journal of Motor Behavior, 41 (4), 305-315.

Vainio, L., Hammare'n, L., Hausen, M., Rekolainen, E., \& Riskila, S. (2011) Motor inhibition associated with the affordance of briefly displayed objects. The Quarterly Journal of Experimental Psychology, 64(6), 1094-1110.

Vainio, L., \& Mustonen, T. (2011). Mapping the identity of a viewed hand in the motor system: evidence from stimulus-response compatibility. Journal of Experimental Psychology: Human Perception and Performance, 37(1), 207-221.

Venneri, A., \& Shanks, M. F. (2004). Belief and awareness: reflections on a case of persistent anosognosia. Neuropsychologia, 42, 230-238.

Vocat, R., Staub, F., Stroppini, T., \& Vuilleumier, P. (2010). Anosognosia for hemiplegia: a clinical-anatomical prospective study. Brain, 133(Pt 12), 35783597.

Vocat, R., Saj, A., \& Vuilleumier, P. (2012). The riddle of anosognosia: does unawareness of hemiplegia involve a failure to update beliefs? Cortex, 49(7): 1771-1781.

Wegner, D. M, \& Wheatley, T. (1999) Apparent mental causation: sources of the experience of will. American Psychologist, 54(7), 480-492.

Wolpert, D. M. \& Kawato, M. (1998). Multiple paired forward and inverse models for motor control. Neural Networks, 11, 1317-1329.

\section{Access brought to you by:}

